\title{
Erdbeben in der Antike
}

\author{
Deutungen - Folgen - Repräsentationen \\ Hrsg. v. Jonas Borsch u. Laura Carrara
}

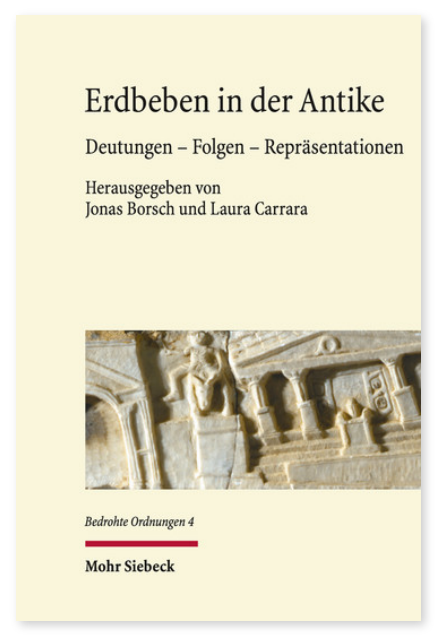

2016. X, 278 Seiten. BedrO 4

ISBN 978-3-16-154170-4 DOI 10.1628/978-3-16-154170-4 eBook PDF 74,00€

ISBN 978-3-16-154169-8

Festeinband $74,00 €$
Der mediterrane Raum gehört weltweit zu den Gebieten mit der höchsten seismischen Aktivität. Insbesondere Griechenland und die heutige Türkei, aber auch große Teile Italiens oder der Levante wurden und werden in regelmäßigen Abständen von Erdbeben heimgesucht, die nicht selten katastrophale Folgen haben. Diese Ereignisse wurden bereits in der antiken Literatur in vielfältiger Art und Weise reflektiert und gedeutet.

Innerhalb der altertumswissenschaftlichen Forschung ist das Thema Erdbeben in den letzten Jahrzehnten zwar verschiedentlich aufgegriffen worden, doch konzentrierte man sich dabei auf einige wenige Aspekte: Im Vordergrund standen die Katalogisierung von Einzelfällen, die Aufbereitung antiker Erklärungsansätze sowie die Untersuchung des materiellen Bewältigungshandelns. Zu vertiefenden Analysen, die das Phänomen über die Jahrhunderte hinweg, in seiner breiten räumlichen Ausdehnung und unter Berücksichtigung der verschiedenen Zeugnisformen betrachten, ist es bisher nicht gekommen.

An dieser Stelle setzt der vorliegende Sammelband an. Er fragt nach den Beziehungen zwischen dem Eintreten von extremen seismischen Ereignissen und zeitgenössischen Weltanschauungen, nach Möglichkeiten und Grenzen der Rekonstruierbarkeit vergangener Bebenkatastrophen sowie nach den Modi ihrer literarischen Repräsentation. Zu diesem Zweck versammelt der Band Expertisen aus unterschiedlichen altertumswissenschaftlichen Disziplinen (Geschichts- und Literaturwissenschaft, Archäologie, Epigraphik) und darüber hinaus (historische Seismologie, Sinologie).

Inhaltsübersicht

Jonas Borsch/Laura Carrara: Zwischen Natur und Kultur: Erdbeben als Gegenstand der Altertumswissenschaften. Eine Einleitung - Emanuela Guidoboni: Erdbeben und Seebeben im antiken Mittelmeerraum: Ansätze für einen Dialog zwischen Seismologie und Geschichtswissenschaft

\section{Deutungen}

Ulrike Ehmig: Der »Erdbebengott Neptun« und die »unbestimmten Erdbebengötter « in lateinischen Inschriften - Stefano Conti: Ende des Herrschers - Ende der Welt? Naturkatastrophen und der Tod des Kaisers - Gerhard Waldherr: Erdbebenkatastrophen bei christlichen Autoren der Spätantike

\section{Folgen}

Wolfram Martini: Schadensbilder. Archäologische Dokumentation von Erdbeben im Mittelmeerraum - Richard Posamentir: Erdbeben als Ende und Anfang: Auflösungsprozesse im römischen Reich - Dora Katsonopoulou: Natural Catastrophes in the Gulf of Corint, northwestern Peloponnese, from Prehistory to Late Antiquity: The Example of Helike - Philipp Deeg: Nero und die Naturkatastrophen - Was der kaiserliche Umgang mit Desastern über Herrschaftsauffassung verraten kann - Christian Fron: Ein Unglück als Chance begreifen lernen. Aelius Aristides' Wirken im Kontext der Erdbeben auf Rhodos und in Smyrna

\section{Repräsentationen}

Claudia Wiener: ratio terrorem prudentibus excutit - Zur Methode und Zielsetzung der Evaluierung von Erdbebentheorien in Senecas Naturales quaestiones - Antje Wessels: Sicherheit im Angesicht der Katastrophe - Zu Senecas Schrift De terrae motu - Giusto Traina: Trajan and the Earthquake of Antioch (115 AD) - Carlo Franco: Ein Erdbeben, ein Rhetor, eine Tradition: Libanios und Nikomedia - Justine Walter: terrae motus und dizhen (地震) - Alles anders am anderen Ende der Welt? Vergleichende Betrachtungen zum Umgang mit Erdbeben in Geschichtswerken aus dem Römischen Reich und dem Alten China

Jonas Borsch Geboren 1984; Studium der Altertumswissenschaften in Trier und Tübingen; 2015 Promotion (Alte Geschichte); Wissenschaftlicher Mitarbeiter am Sonderforschungsbereich 923 »Bedrohte Ordnungen« der Universität Tübingen; seit 2015 Postdoc bei der Heidelberger Akademie der Wissenschaften im Kommentarprojekt zur Chronik des Johannes Malalas.

Laura Carrara Geboren 1984; Studium der Klassischen Philologie an der Universität Pisa und der Scuola Normale Superiore di Pisa; Promotion an der Universität Ca'Foscari Venedig; 2011-15 Wissenschaftliche Mitarbeiterin am SFB 923 »Bedrohte Ordnungen« der Universität Tübingen; seit 2015 Wissenschaftliche Mitarbeiterin im Projekt »Historisch-Philologischer Kommentar zur Chronik des Johannes Malalas« der Heidelberger Akademie der Wissenschaften.

Jetzt bestellen:

https://mohrsiebeck.com/buch/erdbeben-in-der-antike-9783161541704?no_cache=1

order@mohrsiebeck.com

Telefon: +49 (0)7071-923-17

Telefax: $+49(0) 7071-51104$ 